\title{
Oceanic Wind and Balanced Pressure-Height Fields Derived from Satellite Measurements
}

\author{
Roy M. Endlich, Daniel E. Wolf, Christopher T. Carlson and Joseph W. Maresca, Jr. \\ SRI International, Menlo Park, CA 94025
}

(Manuscript received 5 December 1980, in final form 27 April 1981)

\begin{abstract}
Seasat-A scatterometer system (SASS) measurements of wind speed and direction and GOES-2 satellite measurements of cloud motion directions were used to analyze the surface wind field over part of the northern Pacific Ocean for 17 July and 3 October 1978. Surface pressure fields were computed from the nondivergent SASS-derived wind velocity fields using the balance equation and were compared to the National Meteorological Center surface pressure fields. Agreement between the balanced pressure-height fields and National Meteorological Center pressure fields was good. The linear correlation coefficient between the two fields was 0.91 for $17 \mathrm{July}$, and 0.84 for 3 October 1978 . These results indicate that the surface wind and pressure fields of the global oceans (excluding a narrow equatorial zone where the balance equation is invalid) can be determined with acceptable accuracy by using satellite measurements exclusively.
\end{abstract}

\section{Introduction}

Knowledge of the wind and pressure fields at the ocean surface is needed for a variety of meteorological and oceanographic applications. For example, the prediction of surface waves and currents requires wind information over large ocean areas, and numerical weather prediction models require sea level pressure analyses. Also, computation of pressureheight data in the upper atmosphere from vertical temperature profiles measured by satellites requires an accurate knowledge of sea-level pressure. Until recently, ship reports of pressure or wind were the primary source of in situ data. Unfortunately, these reports are sometimes unreliable and are concentrated along shipping lanes, leaving many areas unreported. Grid-point analyses over the oceans are usually obtained by first computing the surface pressure field from the available ship reports to obtain the required coverage and then computing the surface geostrophic wind speed and direction from the pressure field.

Oceanic wind observations increased dramatically during the lifetime of Seasat-A. The Seasat microwave scatterometer system (SASS) obtained global measurements of surface wind speed and direction accurate to $2 \mathrm{~m} \mathrm{~s}^{-1}$ and $20^{\circ}$ (Jones et al., 1979; Barrick and Swift, 1980). These measurements provided the required spatial coverage to map the surface wind field accurately over large ocean regions. The SASS geophysical algorithm seldom gives a single wind direction; instead it gives as many as four directions. Additional information is needed to select the proper one. $\mathrm{Yu}$ and $\mathrm{McPherson}{ }^{1}$ described a method for computing the surface pressure field from SASS wind measurements. They resolved the directional ambiguities by using ship reports and previous National Meteorological Center surface pressure analyses. Their method combined the SASS wind data geostrophically with all available surface reports of pressure and wind. The SASS data influenced their analyses to an extent inversely proportional to the amount of conventional data; i.e., SASS data had greatest influence in areas of sparse conventional reports.

This paper describes a method for computing sea level wind and pressure fields entirely from remote measurements. The surface wind field is obtained from Seasat-A; the ambiguities of direction are resolved using wind direction estimates made from Geostationary Operational Environmental Satellite (GOES)-2 cloud images. Initially, we intended to use the wind direction estimates made from the Wide Aperture Research Facility (WARF) high-frequency (HF) skywave radar operated by SRI International; however, several planned experiments coordinated between Seasat-A and the WARF were never conducted because of the short lifetime of the satellite. Nevertheless, a small amount of coincident data obtained during other experiments is presented in Section 5.

\footnotetext{
${ }^{1}$ Yu, T., and R. D. McPherson, 1979: Surface pressure analysis using scatterometer-derived wind data from the Seasat-A satellite. Preprints Fourth Conference Numerical Weather Prediction, Silver Spring, Amer. Meteor. Soc., 351-355.
} 
It is well known that the simple geostrophic relationship can be used to compute winds from pressure, or conversely, the pressure field from winds. However, numerical weather prediction models require complete fields of both wind and pressure that are "balanced." 'There are different methods for using wind and pressure observations to achieve the required balance. For larger-scale weather phenomena, the traditional approach requires pressure analyses and computes balanced winds from the pressure field (e.g., Shuman, 1957). In the opposite approach, which is primarily concerned with smaller scale phenomena, the balanced pressure field is computed from the wind field (e.g., Anthes and Keyser, 1979). We have used this latter method, which is computationally the simpler of the two.

We analyzed SASS wind measurements for two typical oceanic meteorological cases. We computed wind vectors on a regular grid mesh, made them nondivergent, computed the balanced pressure-height fields from these winds, and compared the balanced pressure heights to NMC pressure analyses. Agreement was good, as shown by the linear correlation coefficients between the two fields for the two cases: 0.91 and 0.84 .

\section{SASS and GOES wind data}

SASS observations in the northeastern Pacific Ocean were obtained from the Environmental Data Service of the National Oceanic and Atmospheric Administration (NOAA) for 16-17 July 1978, and 3-4 October 1978. The July period was selected because well-defined high- and low-pressure systems could be identified in the northern Pacific Ocean, and also because some coincident WARF skywave radar data were available. The October period also had distinct synoptic weather patterns. The SASS

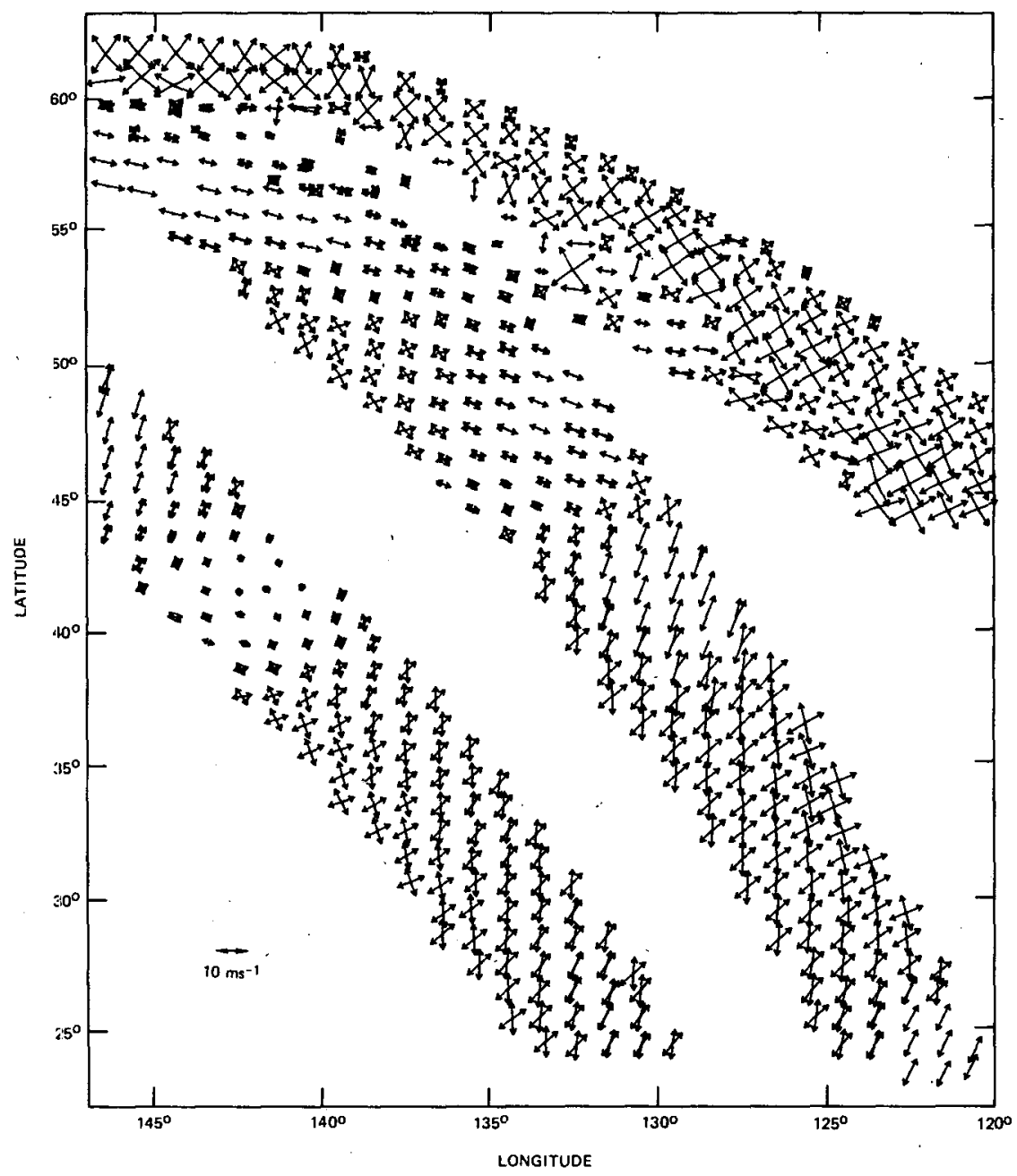

FIG. 1. Plot of sea surface wind data measured by the Seasat scatterometer system (SASS) in the northeastern Pacific on 17 July 1978 showing directional ambiguities. This represents a portion of the total data analyzed. 


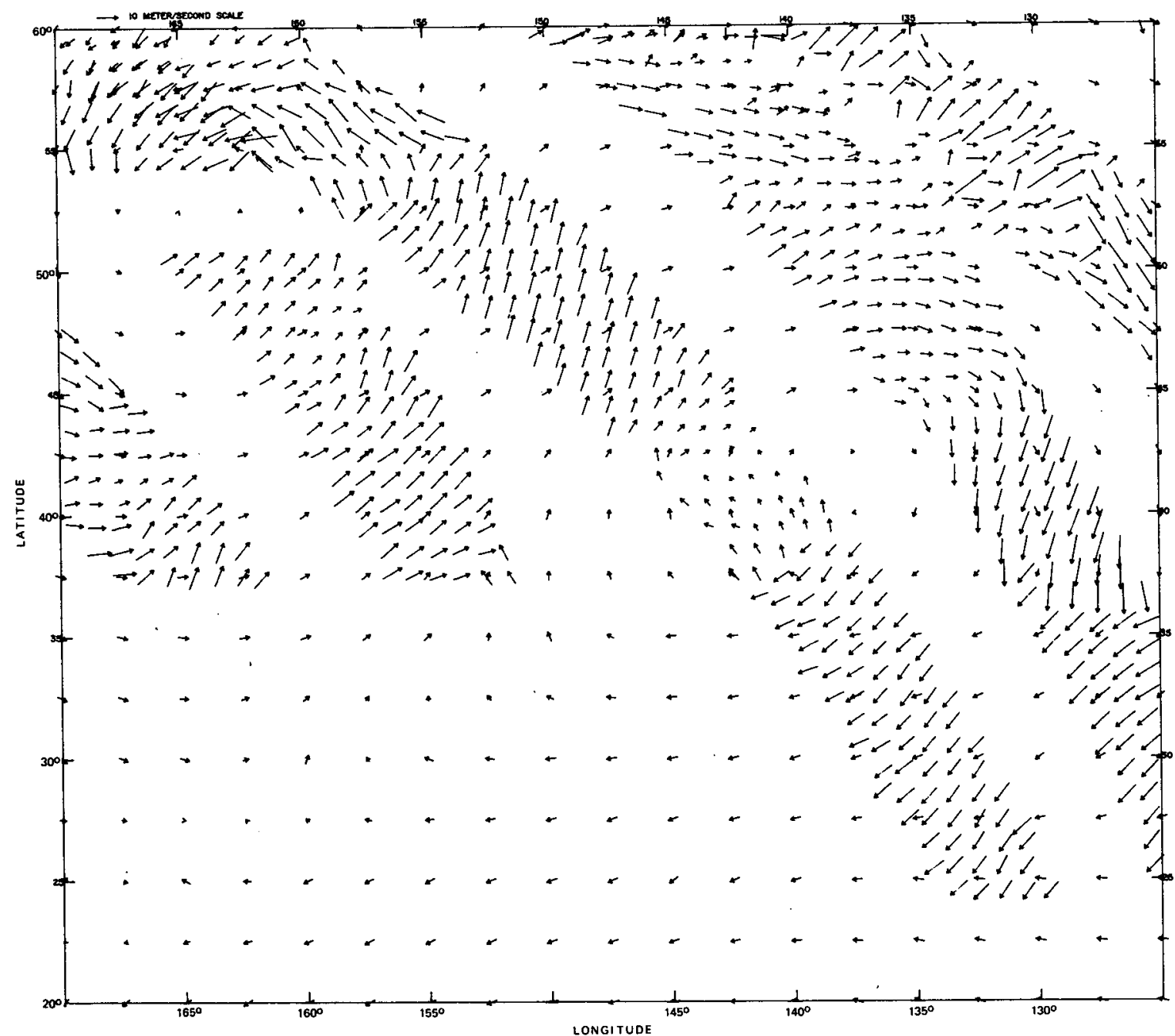

FIG. 2. Plot of low-level cloud motion directions from the GOES satellite analyzed on a $2.5^{\circ}$ grid (short arrows) and SASS winds (longer arrows) selected to be in best agreement, with the cloud motion directions.

surface wind data are computed from an algorithm that relates microwave scattering from ocean capillary waves to wind speed and direction (Jones et al., 1979). An example of the SASS wind speed and direction data for part of the northern Pacific Ocean between 1212 and 1554 GMT on 17 July 1978 is shown in Fig. 1. Up to four ambiguous wind direction vectors exist for many of the SASS data points. The length of each arrow is proportional to speed; however, the computed SASS speed is only a weak function of direction. For wind direction analysis we obtained film loops of the infrared (IR) images from the National Climatic Center taken at $30 \mathrm{~min}$ intervals by the western GOES-2 satellite.

\section{Wind direction estimates from GOES-2 satellite images}

To resolve the SASS directional ambiguity, we used estimates of the wind direction in the lower atmosphere. Grid-point analyses were made of the low-cloud directions. Cloud motions can be computed by experienced analysts through visual study of GOES-2 image sequences using electronic timelapse viewing devices (Serebreny et al., 1970; Smith, 1975). Cloud motions also can be calculated automatically from digital data by cross correlation (Leese et al., 1971) or by using pattern recognition techniques (Wolf et al., 1977). If one requires only the cloud motion direction, it is simple and efficient to view the GOES-2 film loops using a standard movie projector. We determined the direction of cloud motions at intervals of $5^{\circ}$ latitude and longitude using this latter method. The accuracy of the directions decreased poleward of $50^{\circ}$ latitude due to the obliquity of the GOES viewing angle; nevertheless, for the present application the results are satisfactory. We assigned the cloud-height class in the categories low, middle and high, based on the brightness of the 
IR image. Low-cloud motions were used for resolving the ambiguities of SASS directions. However, in certain regions (such as fronts, jet streams, and the intertropical convergence zone) where the satellite film loops show middle and high clouds, but not low clouds, lower level winds may be estimated through general knowledge of atmospheric structure. Of course, in some regions there are no clouds at all, and no cloud-motion entry is made.

In the next step we used an objective analysis technique (Mancuso and Endlich, 1973) that interpolates the cloud motion directions onto a $2.5^{\circ}$ latitude-longitude grid. In this technique, a grid-point value of a meteorological quantity (such as the wind component towards east or north) is computed from several nearby observations. Each observation is given a weight inversely proportional to its distance from the grid point. Also, because of the streaky nature of flow patterns, observations located in an upwind or downwind direction from a grid point are given greater weight than cross-stream observations at the same distance. This method interpolates smoothly between the available observations. In analyzing cloud-motion directions, a nominal speed of $5 \mathrm{~m} \mathrm{~s}^{-1}$ was assigned to each cloud-motion observation because the objective analysis method for winds requires a vector value at each grid point. Fig. 2 shows the cobjective analysis of cloud-motion directions for $17 \mathrm{July}$ on the $2.5^{\circ}$ grid.

To use the low-level cloud-motion directions as a basis for selecting the most likely SASS-derived surface direction, it is necessary to correct for the rotation of wind direction with height in the atmospheric boundary layer. This vertical wind-direction variation is described by the well-known Ekman spiral. The exact nature of this variation at a particular location place depends on such factors as wind speed, thermal stability, and horizontal temperature gradients (thermal winds). For the present analyses, we assumed that the directional turning is everywhere a constant angle. By experimenting with actual data to get the best overall agreement between cloud motions and SASS directions, we determined that $\sim 10^{\circ}$ gave optimal agreement. Therefore, all cloud-motion directions were turned $10^{\circ}$ in a counterclockwise direction before being used to select a SASS direction.

\section{Selection of SASS directions}

The algorithm which selects the SASS direction in best agreement with the corrected cloud-motion direction is similar in concept to meteorological objective analysis methods. Each SASS observation is compared to a direction $\theta$ which is computed as a weighted average of the four nearest cloud-motion directions. In this computation of $\theta$, the weight given to each cloud-motion direction is inversely propor-

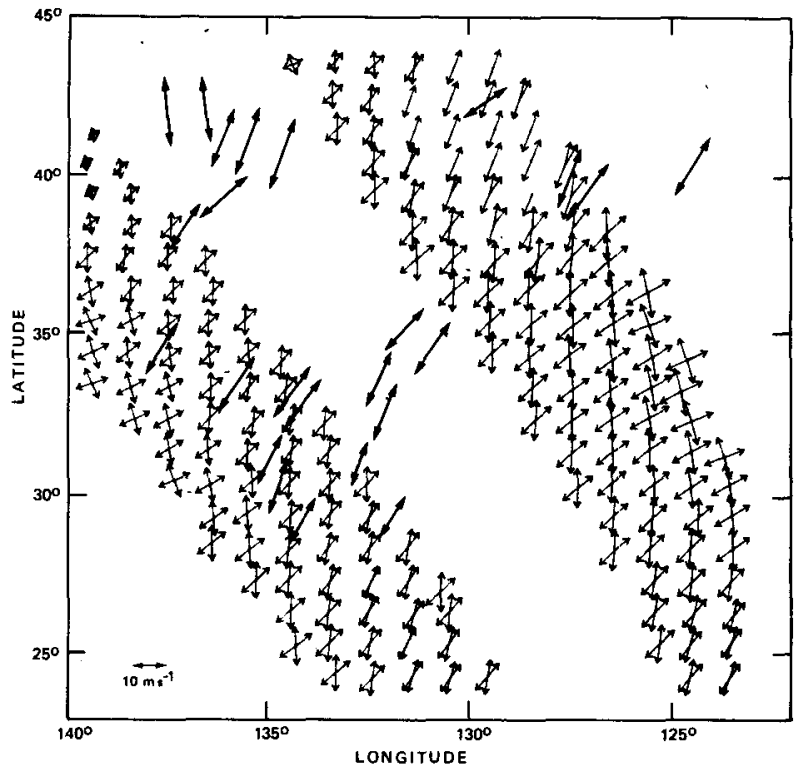

FIG. 3. Plot of SASS wind data for the southern part of Fig. 2 along with corresponding wind directions determined by the WARF skywave radar (heavy arrows).

tional to its distance from the SASS observation. As mentioned previously, to allow for directional turning with height, the SASS direction in closest agreement with $\left(\theta-10^{\circ}\right)$ is selected for subsequent analysis. Fig. 2 shows the SASS directions selected from Fig. 1. (Note that the area covered in Fig. 2 is much larger than the area of Fig. 1.) In only a few instances (out of approximately 850 decisions) were the directional selections different than we would have made subjectively given the same information.

\section{SASS wind direction estimates from HF skywave radar data}

Surface wind direction estimates, accurate to within $16^{\circ}$, can be made by using the WARF HF skywave radar (Stewart and Barnum, 1975). Using methods described in Stewart and Barnum (1975), Maresca and Barnum (1979), and Maresca and Carlson (1980), estimates of wind direction were derived from the radar measurements of short ocean wind waves ( $\sim 10 \mathrm{~m}$ long) propagating toward and away from the radar. These short gravity waves are closely coupled to the winds and therefore represent a good estimate of the surface wind direction. A left-right directional ambiguity exists relative to the radar beam and may be resolved with knowledge of the general circulation in the region of measurement.

An example of WARF and SASS data with their respective ambiguities is shown in Fig. 3. The WARF data cover the $8 \mathrm{~h}$ time span from approximately 1600 to 2400 GMT, whereas the SASS data were recorded slightly earlier (1200-1600 GMT). In the area of overlap, most of the SASS vectors are either 
oriented approximately $360-180^{\circ}$ or $30-210^{\circ}$, and the WARF directions indicate winds are predominantly from about $30^{\circ}$ or $140^{\circ}$. Thus, in general, there is only one wind direction in common between the WARF and SASS, namely, that from the northnortheast. This illustrates how the WARF data may be used to resolve the SASS ambiguities. As mentioned previously, however, the short lifetime of Seasat prevented a coordinated experiment that would have given a dense network of WARF data to compare with SASS data. Figs. 2 and 3 illustrate that in the area of WARF data (the southeastern part of Fig. 2) the SASS directions selected using cloud motions also are from the north or northeast. Thus, the different types of data are in good qualitative agreement.

\section{Examples}

\section{a. Data for 17 July 1978}

The SASS wind vectors for 17 July 1978 (shown in Fig. 2) were analyzed on a $2.5^{\circ}$ grid shown in Fig. 4. The SASS and cloud-motion data are not error-free and, therefore, errors are introduced in the objective analysis; however, the spatial continuity of the analyzed SASS vectors of Fig. 4 indicates that the error level is typical of low-level wind reports. A convenient way to eliminate a large amount of error is to decompose the wind field into nondivergent and divergent parts, and to discard the divergent portion. This is a common meteorological practice, since divergent wind components are usually relatively small compared to their counterparts.

Figs. $5 \mathrm{a}$ and $5 \mathrm{~b}$ show the nondivergent and di-

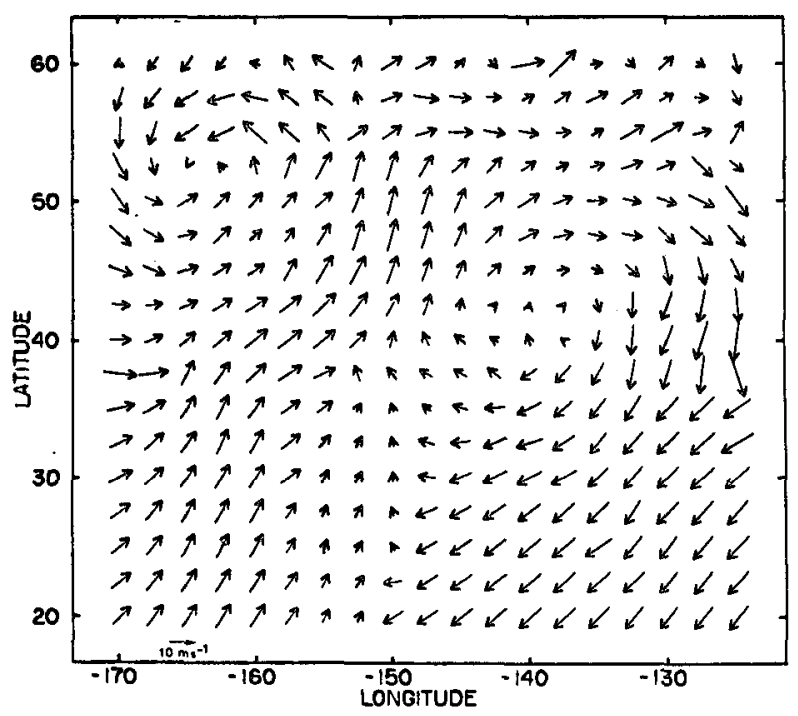

FIG. 4. Objective analysis of the SASS sea surface winds shown in Fig. 2. (The analysis in the southwestern portion is an outward extrapolation from the available data.)
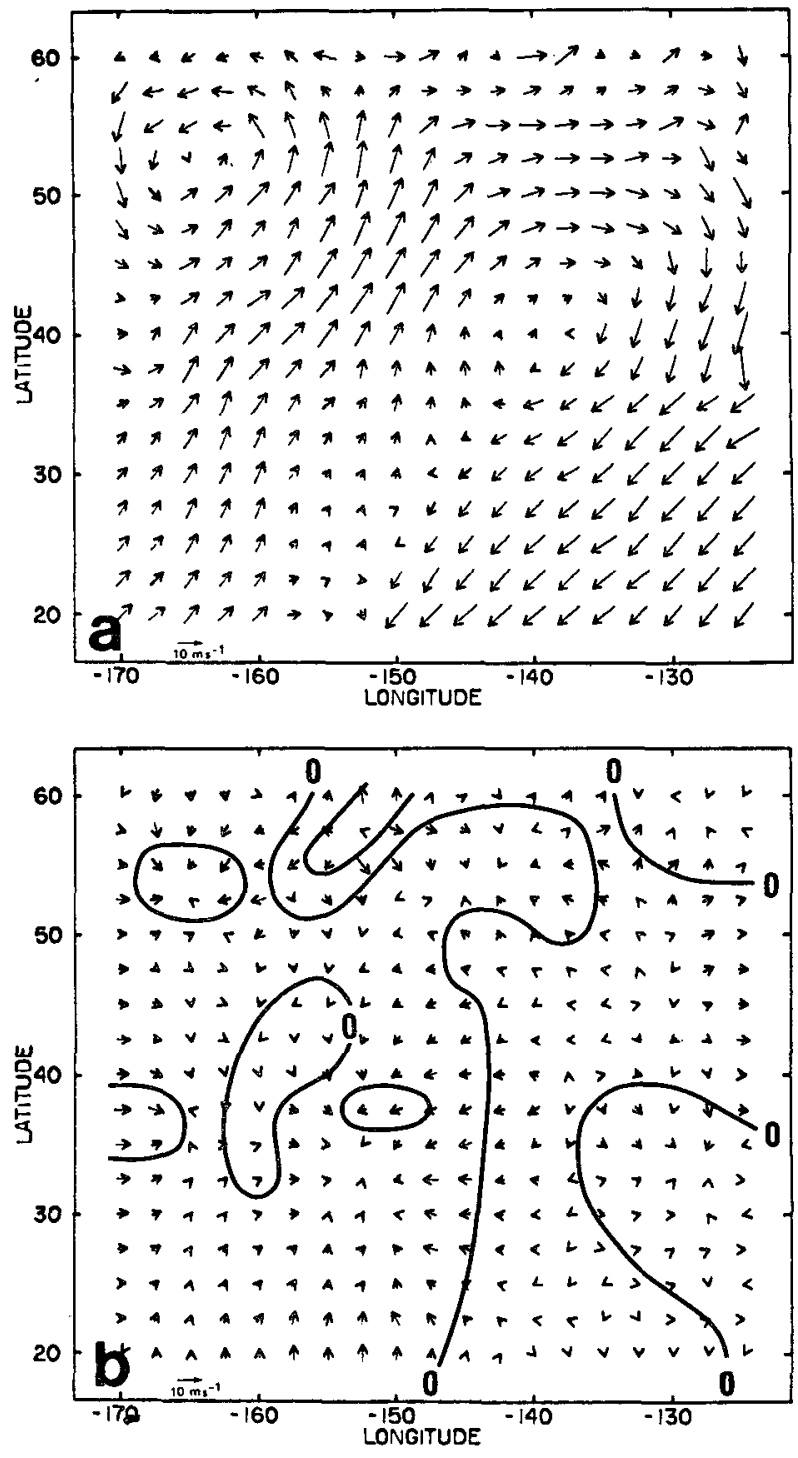

FIG. 5. Plot of wind fields corresponding to Fig. 4. (a) Nondivergent vectors, and (b) divergent (irrotational) vectors with divergence isolines for 0 and $\pm 2 \times 10^{-5} \mathrm{~s}^{-1}$.

vergent vectors corresponding to Fig. 4. The computation was made using the method of direct vector alterations (Endlich, 1967) and does not require computation of a streamfunction and a potential function. The isolines of divergence in Fig. $5 \mathrm{~b}$ are for values of 0 or $\pm 2 \times 10^{-5} \mathrm{~s}^{-1}$. There is a center of convergence associated with the low pressure center; otherwise the divergence patterns appear to be randomly distributed within the wind field which indicates that they are predominantly noise. The nondivergent vectors are smoother (i.e., have less smallscale, random variability) than the total wind vectors of Fig. 4. Also, Fig. 5b shows that the divergent vectors are generally quite small (mostly $<1 \mathrm{~m} \mathrm{~s}^{-1}$ ), and we believe it is reasonable to discard them. 
Their small magnitude is evidence that the SASS speed measurements meet the design accuracy.

The relationship of nondivergent winds to the pressure field is given by the well-known balance equation: In its simplest form (saving only the two largest terms), this equation reduces to the geostrophic relationship. However, it is preferable to use a more general form than the geostrophic one; a typical form is

$$
g \nabla^{2} z=f \zeta-\beta u+2 J(u, v),
$$

where $z$ is the height of a pressure surface, $f$ is the Coriolis parameter, $\zeta$ is the relative vorticity, $\beta$ $=\partial f / \partial y$, and $J(u, v)=(\partial u / \partial x)(\partial v / \partial y)-(\partial u / \partial y)$ $\times(\partial v / \partial x)$. This form can be used either to compute the balanced height field or to compute the geostrophic wind vectors directly from the nondivergent winds. The balanced pressure-height field that corresponds to Fig. $5 \mathrm{a}$ is computed by relaxation from (1) and is shown un Fig. 6a. We ușed an alternating direction implicit method of solution and normal gradient (Neumann) boundary conditions (Mancuso, 1967). There is a distinct low center at $52^{\circ} \mathrm{N}, 165^{\circ} \mathrm{W}$, and a high at $40^{\circ} \mathrm{N}, 139^{\circ} \mathrm{W}$. In the southwestern part of the area there are no SASS observations, so the analyzed fields are an outward extrapolation from the available data and lose reliability with distance. The original computations of balanced pressure height were made in units of height $(\mathrm{m})$ but have been converted to pressure (mb) for comparison to the NMC sea-level pressure analysis for 1200 GMT (Fig. 6b). The transformation from height to pressure was made by assigning corresponding heights and pressures at two points, one in the high and one in the low. The linear correlation coefficient between Figs. $6 \mathrm{a}$ and $6 \mathrm{~b}$ is 0.91 , and the rms difference between them is $4.4 \mathrm{mb}$. This difference is due both to uncertainties in the analyses and to time differences of a few hours.

The correlation coefficient is based on 260 pairs and would have a $99 \%$ probability of lying between 0.87 and 0.94 for independent data. However, the Seasat and NMC analyses are not entirely independent because NMC analyses use a variety of data including cloud motion vectors. The influence of the cloud motions in NMC analyses is difficult to determine since it depends on the amounts and locations of all data vithin the area of interest. Thus, we conclude that the true correlation between our Seasat analysis and the NMC analysis is somewhat less than that indicated above.

\section{b. Data for 3 October 1978}

Similar procedures were followed for data of 3 October 1978. The Seasat and GOES-2 observations cover the portion of the northern Pacific Ocean shown
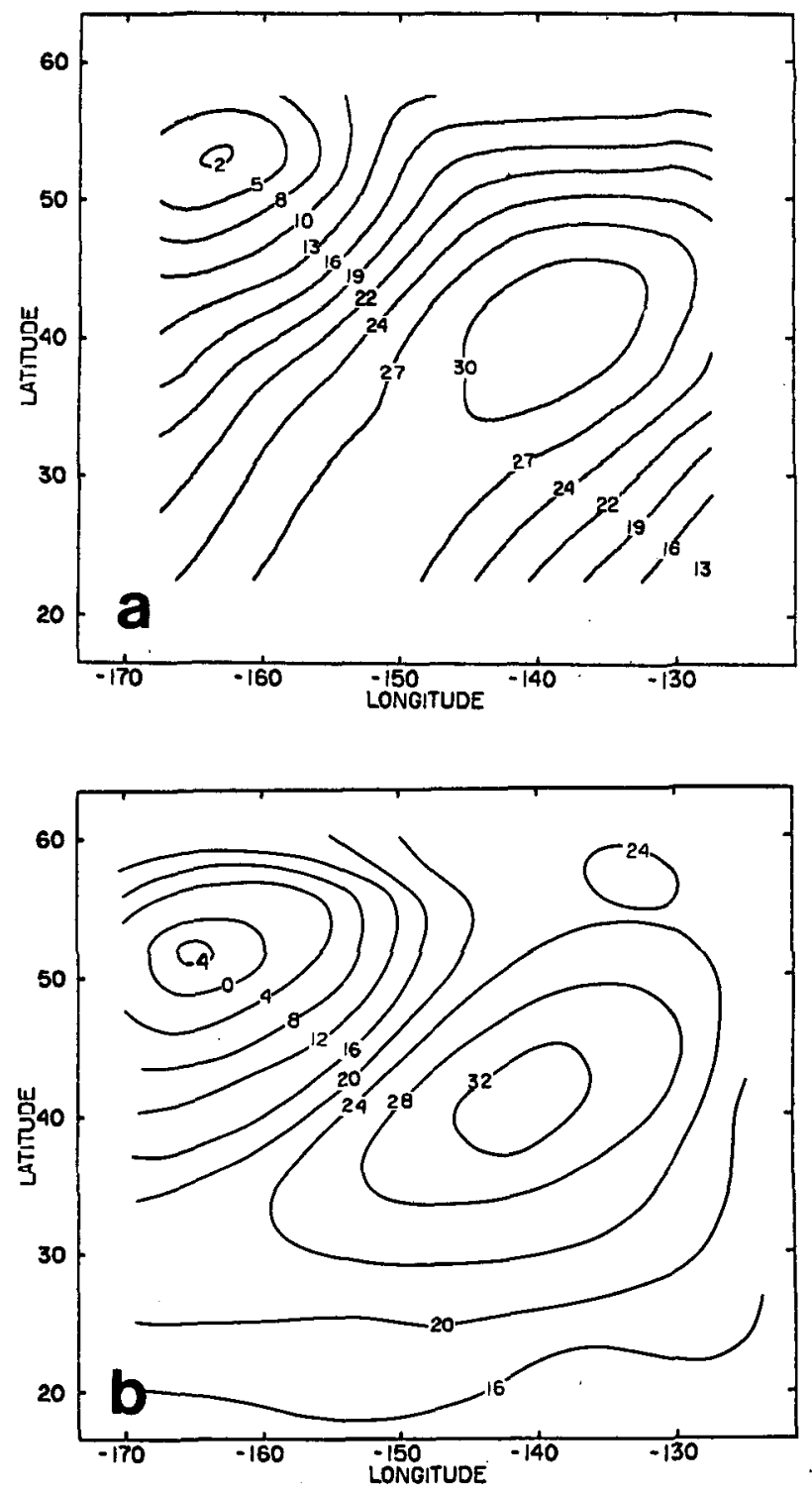

FIG. 6. Plot of pressure fields (departures from $1000 \mathrm{mb}$ ) corresponding to Fig. 4. (a) Balanced values computed from the winds of Fig. Sa, and (b) corresponding NMC analysis (for 1200 GMT 17 July 1978).

in Fig. 7. When computing the balanced height from (1) for data in the equatorial region, one must avoid the singularity due to the vanishing of the Coriolis parameter at zero degrees latitude. We assumed that within $5^{\circ}$ of the equator, $f$ retained its $5^{\circ}$ latitude value.

The balanced pressure-height field is shown in Fig. 7a, and the corresponding National Meteorological Center sea level pressure field, transposed to the same grid, is shown in Fig. 7b. The agreement is good except in regard to the location of the lowpressure center in the northwest part of the area. 

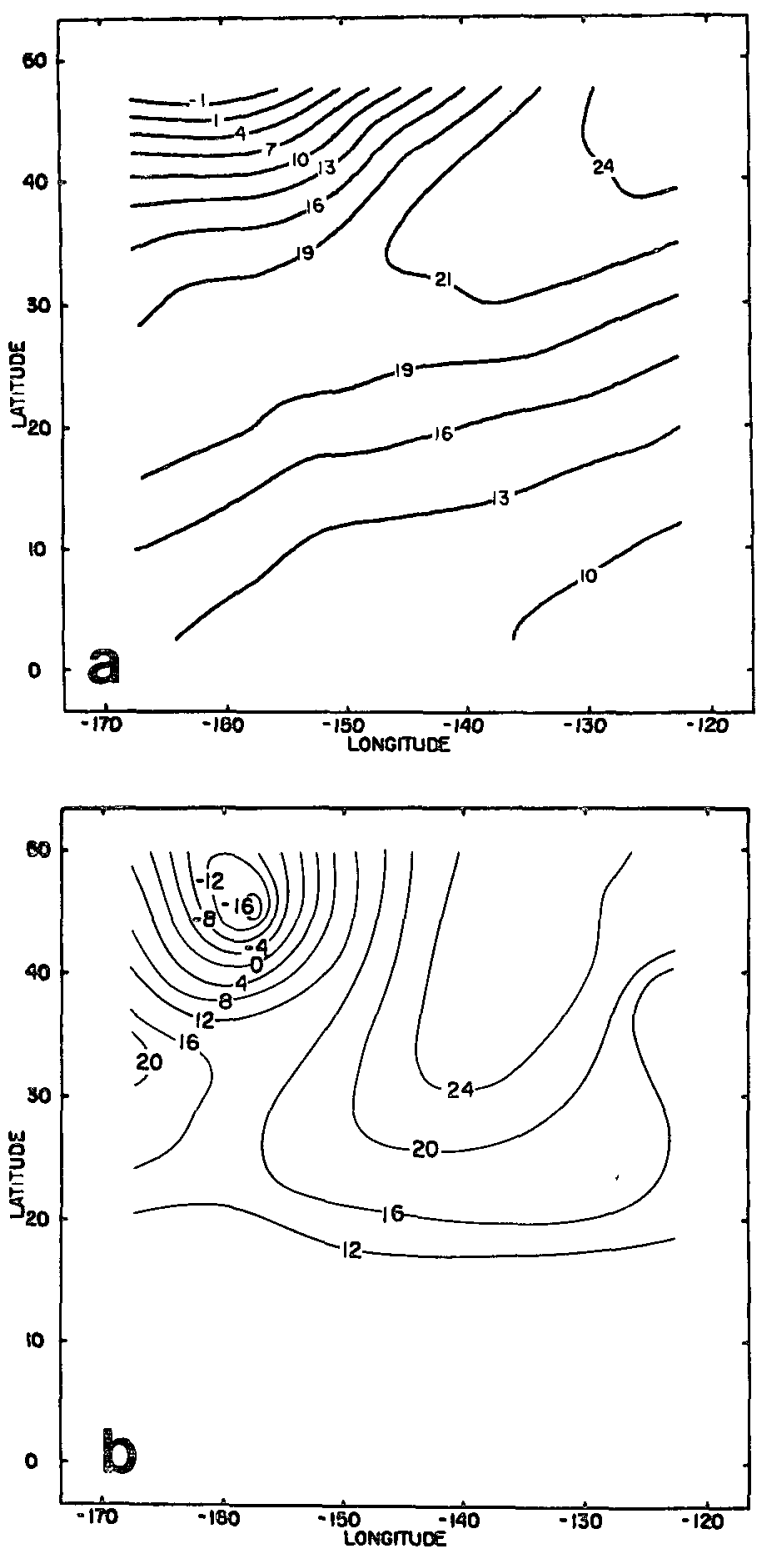

FIG. 7. Plot of pressure fields (departures from $1000 \mathrm{mb}$ ) for 3 October 1978. (a) Balanced values computed from SASS winds for approximately $2100 \mathrm{GMT}$, and (b) NMC analysis ior $1800 \mathrm{GMT}$.

The National Meteorological Center analysis is for $1800 \mathrm{GMT}$, whereas the SASS data in that part of the region are for approximately $2100 \mathrm{GN}$ iT. The GOES-2 film loop shows that the cyclone center was moving northward very rapidly. Therefore, we believe that the disagreement between the locations of the low center in Figs. $7 \mathrm{a}$ and $7 \mathrm{~b}$ is due mainly to the time difference. The linear correlation coefficient between the balanced-pressure field and the National Meteorological Center pressure field is 0.84 which seems large in the light of the visual differences in the patterns. It is evidently due to their large scale similarities. For 250 pairs the correlation coefficient would have a $99 \%$ probability of lying between 0.76 and 0.88 for independent data. The rms difference between the two fields is $5.4 \mathrm{mb}$.

\section{Conclusion}

We computed the balanced-pressure field over the Pacific Ocean from data obtained solely from remote sensors. We compared these balanced-pressure fields computed from the SASS surface wind velocity measurements to National Meteorological Center analyses for two different days. The agreement between these analyses was very good on one day and fair on the second. The correlation coefficients for the two days were 0.91 and 0.84 , with rms differences of 4.4 and $5.4 \mathrm{mb}$. This study suggests that SASS data in combination with other remote sensor data, such as those provided by geosynchronous satellites or skywave radar, can be used to measure sea level winds and to compute the balanced-pressure fields.

We wish to point out that all procedures discussed earlier can be automated; for example, the cloudmotion tracking can be performed in most cases by digital pattern recognition techniques, and algorithms can be developed for resolution of the wind directional ambiguity. Comparisons should be made of the results of these techniques with other similar results (e.g., Yu and McPherson) ${ }^{1}$ for both the Northern Hemisphere, where conventional data are numerous, and the Southern Hemisphere, where conventional data are sparse.

Acknowledgments. This study was carried out under NOAA Contract NA795AC0073 with Dr. Donald Barrick of the Wave Propagation Laboratory of NOAA, Boulder, Colorado, as Technical Officer. We thank him and also thank Peter Woiceshyn of the Jet Propulsion Laboratory for helpful comments and suggestions.

\section{REFERENCES}

Anthes, R. A., and D. Keyser, 1979: Test of a fine-mesh model over Europe and the United States. Mon. Wea. Rev., 107, 963-984.

Barrick, D. E., and C. T. Swift, 1980: The Seasat microwave instruments in historical perspective. IEEE J. Oceanic Eng., OE-5, 74-79.

Endlich, R. M., 1967: An iterative method for altering the kinematic properties of wind fields. J. Appl. Meteor., 6, 837-844.

Jones, W. L., P. G. Black, D. M. Boggs, E. M. Bracalente, R. A. Brown, G. Dome, J. A. Ernst, I. M. Halberstam, J. E. Overland, S. Peteherych, W. J. Pierson, F. J. Wentz, P. M. Woiceshyn and M. G. Wurtele, 1979: Seasat Scatterometer: Results of the Gulf of Alaska workshop. Science, 204, 1413-1415. 
Leese, J. A., C. S. Novak and B. B. Clark, 1971: An automated technique for obtaining cloud motion from geosynchronous satellite data using cross correlation. J. Appl. Meteor., 10, $118-132$.

Mancuso, R. L., 1967: A numerical procedure for computing fields of streamfunction and velocity potential. J. Appl. $\mathrm{Me}$ teor., 6, 994-1001.

-, and R. M. Endlich, 1973: Wind editing and analysis program-Spherical grid (WEAP-1A). User's Manual, Contract DAHC04-71-C-0013, Stanford Research Institute, Menlo Park, CA, 47 pp.

Maresca, J. W., Jr., and J. R. Barnum, 1979: Remote measurement of the position and surface circulation of Hurricane Eloise by HF skywave radar. Mon. Wea. Rev., 107, 16481652.
—_, and C..T. Carlson, 1980: High-frequency skywave radar measurements of Hurricane Anita. Science, 209, 1189-1196.

Serebreny, S. M., E. J. Weigman, R. G. Hadfield and W. E. Evans, 1970: Electronic system for utilization of satellite cloud pictures. Bull. Amer. Meteor. Soc., 51, 848-855.

Shuman, F. G., 1957: Numerical methods in weather prediction: I. The balance equation. Mon. Wea. Rev., 85, 329-332.

Smith, E., 1975: Man-computer interactive data access system. IEEE Trans. Geosci. Electron., GE-13, 123-126.

Stewart, R. H., and J. R. Barnum, 1975: Radio measurements of oceanic winds at long ranges: An evaluation. Radio Sci., 10, 853-857.

Wolf, D. E., D. J. Hall and R. M. Endlich, 1977: Experiments in automatic cloud tracking using SMS-GOES data. J. Appl. Meteor., 16, 1219-1230. 